\title{
The Active Video Game Paradox
}

\author{
Sam Kirk ${ }^{1}$, Andrew J. Manley ${ }^{1}$ \\ ${ }^{1}$ Leeds Beckett University, Carnegie School of Sport, UK, E-mail \\ s.kirk@leedsbeckett.ac.uk
}

\begin{abstract}
Evidence shows inactivity and obesity are a substantial global burden, economically and physiologically. Sedentary screen time strongly contributes to this burden. Sedentary screen time is often achieved through video game play. Active video games (AVGs) tried to solve the screen time problem. AVGs work, having small to moderate positive effects on activity levels and body composition changes. However, they perform consistently poorly on the commercial market. This represents a paradox: AVGs work pragmatically, yet underperform commercially, limiting their impact practically. The purpose of this study was to explore this paradox with the aim of understanding how AVGs compare to non-active video games (NAVGs), based on gameplay experience. Findings revealed that AVGs have a significant negative comparison to NAVGs. Qualitative exploration highlighted several important considerations; gamer motivation, the lack of quality and variety in AVGs, the stereotype in AVGs, and the practical limitations of the hardware that supports AVGs. Perception of an augmented reality platform, a potential solution to the issues, was found to be positive.
\end{abstract}

Keywords: Active Video Games, Non-Active Video Games, Gamer Motivation;

\section{Introduction}

The following section infers the paradox of AVGs from macro, general, global findings, filtering level by level to a micro, specific, nuanced issue that requires innovative solutions.

\subsection{Such a burden}

The global cost of inactivity, when considering direct healthcare costs, indirect productivity losses and lifetime disease burden, was estimated at $\$ 67.5$ billion [1]. The study is arguably the most scientifically rigorous investigation to date of the cost estimates of physical inactivity. However, the authors admit, achieving such rigor resulted in conservative financial estimates. The inclusion of additional methods would mean the economical and physiological cost of inactivity is significantly north of $\$ 67.5$ billion. Additional methods would include extra non-communicable diseases for direct healthcare costs and lifetime disease burden [2], [3], absenteeism and sickness for indirect productivity costs [4], [5], and the inclusion of mental health conditions in estimation of the burden [6].

Given the positive correlation that obesity shares with physical inactivity [7], the cost estimates should be considered in this argument as well. McKinsey Global Institute [8] estimates the global cost to be $\$ 2.0$ trillion annually, representing the third largest share of gross domestic product, 5\% lower than smoking and armed conflict. Though the rigor applied, methods used, figures proposed, and dates of publication differ, one theme was evident: physical inactivity and obesity incur a significant cost to the world's population, economically and physiologically. 
pag. 4

\subsection{Sedentary screen-time as technological villain}

Given the scale and scope of the physical inactivity burden, an understanding is required of how sedentary time is being spent. Four systematic reviews [7], [9]-[11] analysed 420 studies regarding sedentary behaviour and adverse health outcomes, such as obesity and mortality. Collectively, the life cycle was considered, from school-aged children [11], to young people [7], to adults [9], [10]. The four systematic reviews present robust evidence that sedentary behaviour, particularly in the form of screen-time upwards of two hours a day, has adverse health outcomes in the short and long-term.

\subsection{Video games and their many faces}

With the evidence showing people are accumulating over two hours of risk-laden screentime per day, an understanding is now needed as to what types of screen-time people engage in. There is a strong statistical argument presented in the video game industry, wherein the global gamer count was estimated at 2.5 billion [12]. UK-based research [13] estimates that $18.8 \mathrm{~m}$ people aged 6-64 play video games for an average of 8.8 hours a week. However, research from UKIE [14] estimates $33.5 \mathrm{~m}$, or 1 in 2 people aged 8-74 in the UK, play video games for an average of 14 hours a week. Fourteen hours over seven days equals, on average, two hours a day, which is the risky upper-limit outlined in the previous paragraph. Collectively, these estimates suggest between $29 \%$ and $51 \%$ of gamers are at risk of adverse health outcomes from video game play.

Video games are traditionally a sedentary activity, wherein a person sits/stands with a controller in their hand and presses buttons to control the characters on the screen. A subcategory of video games, known as AVGs, endeavoured to have user-motion partly, or wholly, replace controller input. The evidence advocating AVGs as an alternative to sedentary screen-time is robust and somewhat positive, showing improvements in body composition in some participants and small-to-moderate increases in physical activity levels, albeit to a low/moderate intensity level [15]-[18]. Despite these promising findings, a downward trend in inactivity and obesity statistics has not been observed [19].

Commercially, market statistics show AVG titles have rarely featured in the top 100 annual commercial sales charts over the past seven years [20]-[26]. This raises questions of quality, as it seems gamers are more motivated to play, and keep playing, traditional NAVGs. When someone wants to play a game, they decide what game to play, since at any one timepoint, they can only play one game. As such, this study compares AVGs to NAVGs on equal grounds, because it is the competition for attention that AVGs need to win to have a greater impact across society, regardless of their pragmatic benefit.

\subsection{Gamer motivation}

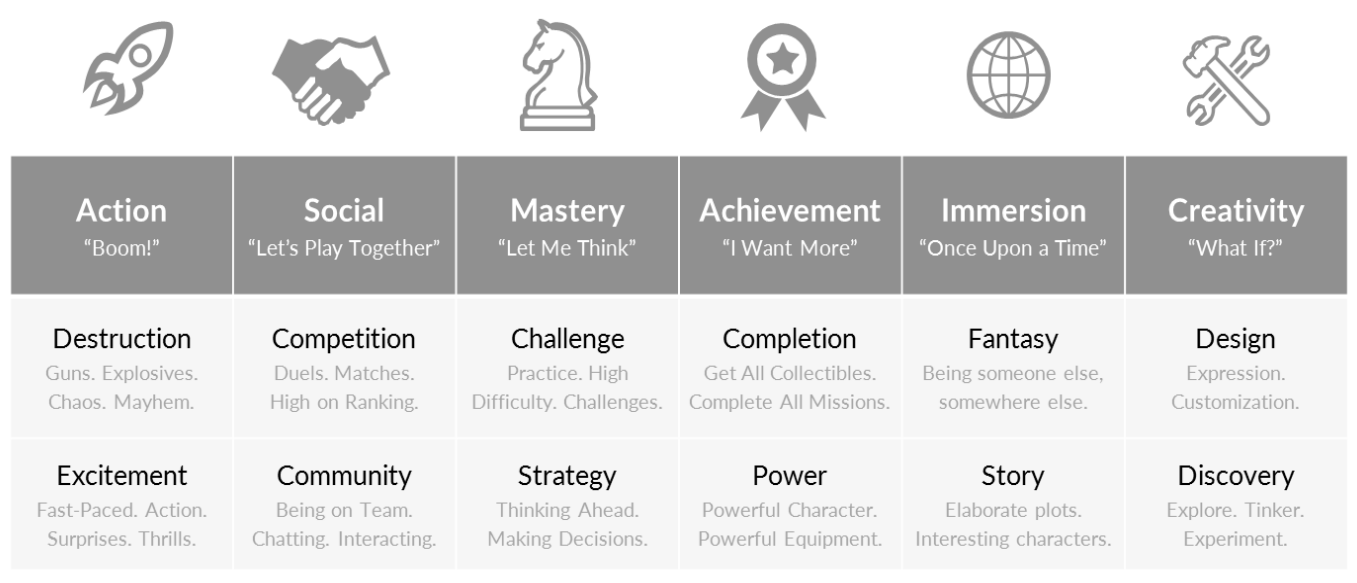

Figure 1. The Gamer Motivation Model [29]. 
Within the last decade, psychology within video games has received greater attention. There are several theories that explore gamers' decisions to start playing and keep playing; this paper addresses two. Self-Determination Theory (SDT) [27] represents a general explanation of human motivation which has been explored within video games. SDT refers to three basic psychological needs of autonomy (a sense of control), competence (a sense of achievement or mastery), and relatedness (a sense of belonging), each having been argued to enhance the video gamer's experience [28]. The Gamer Motivation Model (GMM; see Figure 1) [29] represents a more context-specific model to explain behaviours and motives of gamers. It expands on the body of knowledge accrued to date, adapting research from all facets of media to develop an empirical model of motivation in gamers, based on the responses from over 400,000 participants at the time of writing. Motivation facilitates the gamer's decision to start playing and keep playing. Thus, the science of human behaviour is a vital area to understand in conjunction with the field of AVGs. These two conceptual frameworks provide a theoretical lens through which the findings of this paper can be viewed.

\subsection{The active video game paradox}

Existing evidence shows inactivity and obesity are a substantial global burden, economically and physiologically. Sedentary screen-time strongly contributes to this burden. Sedentary screen-time is often achieved through video game play. AVGs tried to solve the screen-time problem, having small-to-moderate positive effects on activity levels and body composition changes. However, they perform consistently poorly on the commercial market. It is not yet fully understood what makes an AVG successful. What is known is that gamer motivation is an important consideration; market statistics are not the only metric that matters. This lack of understanding is significant, because the learned knowledge could result in fun and innovative ways to improve overall health and wellbeing (e.g., at home for leisure, in medical settings for clinical interventions). The research areas alluded to in this section are fundamentally linked, illuminating the problem which this paper is dedicated to explore: if AVGs work from a utilitarian perspective, why are they not working from a commercial one?

\section{Methods}

\subsection{Study design}

The research problem was best served by a pragmatic, mixed-methods approach with interpretivist leanings. Thus, two stages of data collection were adopted: a quantitative approach (stage 1), and a qualitative approach (stage 2).

Stage 1 aimed to answer the question: How do AVGs compare to NAVGs, when measured on gameplay experience?

Stage 2 aimed to answer the question: Why are AVGs not performing well on the commercial market?

\subsection{Demographic details}

Participants were recruited online, through peer networks via two facilities: a busy leisure centre and a University. Gatekeeper consent was obtained before poster advertisements were used to facilitate recruitment. Participants were all over the age of 18, had knowledge of at least five AVGs, and experience of playing at least one AVG was required. This means it was possible that participants had either played or not played the AVG in question, but certainly had experience of the overall genre. These inclusion criteria were set because the perception of the AVG, whether played or un-played, was equally important as the actual experience in determining an individual's intention to play or keep playing. This study had a convenience sample totalling 32 participants $(n$ male $=25)$, with a mean age of 26.5 years. 
pag. 6

All participants completed stage 1. Stage 2 participants were a sub-sample of stage 1 participants $(n=5)$, of which all were male. Interview length ranged from 47:43 to 84:54 minutes. Participants were asked to provide the name of their favourite video game character, which would serve as a pseudonym throughout the paper.

\subsection{Measures}

\subsection{1: Stage 1.}

To answer the primary research question related to this stage of the study, an understanding of how people compared video games was needed. Thus, a review process was adopted, for a direct comparison.

Given the study's novel line of inquiry, there were no relevant measures to utilise, representing a need to develop one. Using research alluded to prior [28]-[30] and video game design textbooks [31], [32], the considerations that influence a gamer's motivation were collated. In addition, key foci in game design, such as world design, were applied [31].

Ten design constructs were chosen: world design, characters, story, community, expression, challenge, re-playability, engagement, graphics and audio, competitive. Each design construct was rated on a scale of $0-10$ by the participants, based on how the AVGs compared to NAVGs of the same design construct. The NAVGs were chosen by, and individual to, the participants. This means that all NAVGs were rated at the idealistic 10/10 as default. This allowed the authors to accurately assess comparisons between AVGs and NAVGs. All information needed to complete the review process was provided to participants in a review document, which included definitions of each design construct, with popular examples of what games might score a 0 and a 10 for that design construct. This is somewhat subjective as a result of personal opinions pooled with existing review data via Metacritic [33], coupled with the notion that participants would be comparing AVGs to their favourite NAVGs, the idealistic 10/10. Metacritic is a website that searches the internet and collates all available game reviews. Metacritic provides two separate review scores, one as an average score between independent critics and the other as an average score between consumer reviews (members of the Metacritic site). The review document also contains multiple questions, to prompt the participant to think critically about the design construct, thus providing a well-informed review of the AVG in question [34]. The following examples are for the "world design" construct: Can you explore or is it linear? Can you interact with what is around you (climb, speak, smash)? What is the level of detail? Is it immersive? Does the environment change? What do the levels contribute?

Given the volume of games that could be classed as AVGs (inclusive of games/apps for gyms, territorial "serious games" for exploring cultural heritage, location-based games), it was important to narrow the focus to a selective list of five games which represented some of the most popular and/or best performing AVGs on the commercial market. Consequently, a research-based process was applied to streamline the substantial number of commercial titles available. The games included within the present study were: Pokémon Go (largest user install base in category, and critically acclaimed augmented reality approach); Just Dance 2017 (the only game in category to have a large recurring annual user base); Wii Fit and Wii Sports (first in category to launch, and has the best Metacritic review scores from independent and community reviews); Kung Fu Live (positive critic reviews on the game's approach to "narrative"); and Beatsplosion (positive critic reviews on the game's approach to "world design").

\subsection{2: Stage 2.}

To build on stage 1 findings and answer the specific research question, an understanding of the participants' thoughts and feelings regarding AVGs and NAVGs was needed within this stage. Specifically, it was considered appropriate to have some structure to guide the interview, yet some flexibility to be able to bond, probe, and clarify. Thus, semi-structured interviews were employed. 
The interview guide was reflexively developed courtesy of several pilot tests with peers. Different question types facilitated in-depth data collection [35]. First, rapport building questions, such as 'tell me about your experiences with video games, your best and worst moments for example', were used. Second, various open-ended questions (e.g., 'how do you perceive the quality of the world design within AVGs?') were used. Typical probes to open-ended questions would ask participants to compare their thoughts and experiences of AVGs and NAVGs. Towards the end of the interview, a mixed-reality idea was explored as a potential solution to the AVG issues identified through the literature review and early pilot tests. This allowed the study to collect initial data on a potential solution which could be further explored, or discontinued, dependant on the perspectives expressed by participants. The interview concluded with a probe for additional comments.

\subsection{Analysis}

\subsection{1: Stage 1.}

The first step of analysis was to generate means and standard deviations of each game, and then as an overall genre. Second, paired sample T-tests were conducted on the genre mean and each design construct. This was done to identify if AVGs had any relative weaknesses in specific design constructs. Pairwise comparisons were conducted on stage 1 means and public review data for the five games used. Public data were freely available at Metacritic [33], [36-41]. Through this analysis, opinions of consumers and professional critics could be compared to stage 1 data for a more in-depth understanding of AVGs vs NAVGs. Overall, there was insufficient variance in age, sex, or ethnicity to compare sub-groups in analysis.

Outliers were identified through stem and leaf plots. The decision was made to retain outliers, as ratings were subjective. An extreme rating could be representative of a large enough sub-sample to support the long-term success of a game. For example, $1 \%$ of the $33.5 \mathrm{~m}$ UK gamer population [14] is 335,000 gamers.

\subsection{2: Stage 2.}

A thematic analysis was conducted for stage 2 . Given the philosophical position of pragmatism [42]-[46], abductive logic was applied to the analysis to allow for novel concepts to be created, while accounting for existing ideas and constructs to be explicitly acknowledged. The first step was to analyse the entire dataset inductively (i.e., allowing original themes to be created organically without conscious or explicit reference to established theories or models). To do so, Braun and Clarke's [47] six-stage process was followed. Stages four and five [47] were the point at which deductive logic was applied, utilising gamer motivation theories explored in the literature review [27]-[30] to the analysis, to ascertain the best fit for the created themes.

\section{Quantitative Results and Discussion}

\subsection{Quantitative results}

Table 1 shows descriptive statistics for the five AVGs. Despite the inclusion of outliers, mean ratings of games are still perceived as less than 5. Table 2 shows the overall mean of the five games, named the genre mean. These two tables show that in terms of the game play experience, most participants perceived AVGs to be inferior to NAVG.

Table 3 shows the mean for each design construct compared to the genre mean. This highlights specific perceived weaknesses in the AVG genre. A positive value in the mean column shows that the design construct is viewed as worse than the genre mean. The opposite is true for a negative value. Thus, the findings show that the AVG genre delivers 
pag. 8

lower quality "world design" ( $p<0.01)$, "characters" $(p<0.05)$ and "stories" $(p<0.01)$, compared to the perceived quality of the genre.

Table 1. Descriptive statistics for the five video game reviews. Data source is the individual game means per participant.

\begin{tabular}{|c|c|c|c|c|c|}
\hline $\begin{array}{c}\text { Video Game } \\
\text { Title }\end{array}$ & $\mathbf{N}$ & Minimum & Maximum & Mean \pm SD & Variance \\
\hline Pokemon Go & 32 & 2.30 & 8.50 & $4.76 \pm 1.20$ & 1.45 \\
\hline $\begin{array}{c}\text { Wii Fit and Wii } \\
\text { Sports }\end{array}$ & 32 & 1.40 & 6.10 & $4.80 \pm 1.11$ & 1.24 \\
\hline $\begin{array}{c}\text { Just Dance } \\
\text { Kung Fu Live }\end{array}$ & 32 & 1.10 & 8.70 & $3.35 \pm 1.72$ & 2.95 \\
\hline Beatsplosion & 32 & .60 & 8.80 & $3.54 \pm 1.55$ & 2.41 \\
\hline
\end{tabular}

Table 2. AVG genre mean. Data source is the collective game means (5), per participant, as opposed to the individual means in Table 1.

\begin{tabular}{|c|c|c|c|c|}
\hline Genre & N & Minimum & Maximum & Mean \pm SD \\
\hline$A V G$ & 32 & 2.12 & 8.16 & $3.97 \pm 1.16$ \\
\hline
\end{tabular}

\subsection{Quantitative discussion}

There is one key finding worth discussing that pertains specifically to the quantitative findings. It is clear that AVGs are inferior to NAVGs, in the perceptions of these participants (see Table 1 and 2). This provides evidence that may partially explain low sales statistics [20-26]. In contrast, outliers representing positive reviews of AVGs (2/32 or $6.25 \%$ ) could, if representative of the wider population, be a sufficient audience for those games to be deemed a success, in terms of total gamers. For example, $6.25 \%$ of the $33.5 \mathrm{~m}$ UK gamer population [14], is $2.1 \mathrm{~m}$ gamers. This figure would place the game $22^{\text {nd }}$ on the annual game sales chart [24], although, evidently, this is not the case. These two contrasting points suggest that AVGs have two, possibly complementary, critical issues: i) the quality of gameplay experiences, and ii) a lack of variety in available games and types of gameplay experiences (predominantly rhythm games and sports/fitness games). These issues will be explored further below, when both datasets combine to offer a more holistic explanation. 
Table 3. Pairwise comparisons of the mean ratings of each design construct and the AVG genre mean.

\begin{tabular}{|c|c|c|c|c|c|c|}
\hline $\begin{array}{l}\text { Design Construct - } \\
\text { Genre Pairing }\end{array}$ & Mean & $\begin{array}{l}\text { Standard } \\
\text { Deviation }\end{array}$ & $\begin{array}{l}\text { Standard } \\
\text { Error Mean }\end{array}$ & $t$ & $d f$ & $p$ \\
\hline World Design - AVG & -.95 & .71 & .13 & 7.580 & 31 & .000 \\
\hline Character - $A V G$ & -.39 & .83 & .15 & 2.669 & 31 & .012 \\
\hline Story $-A V G$ & -1.49 & .96 & .17 & 8.748 & 31 & .000 \\
\hline Community - AVG & .42 & .74 & .13 & -3.22 & 31 & .003 \\
\hline Expression - $A V G$ & -.07 & .82 & .14 & .510 & 31 & .614 \\
\hline Challenge - $A V G$ & .51 & .52 & .09 & -5.574 & 31 & .000 \\
\hline Replayability - AVG & .50 & .73 & .13 & -3.831 & 31 & .001 \\
\hline Engagement - $A V G$ & .57 & .61 & .11 & -5.574 & 31 & .000 \\
\hline $\begin{array}{c}\text { Graphics \& Audio - } \\
A V G\end{array}$ & .49 & .63 & .11 & -4.416 & 31 & .000 \\
\hline Competitive - AVG & .41 & 1.12 & .20 & -2.094 & 31 & .045 \\
\hline
\end{tabular}

\section{Qualitative Results and Discussion}

Four higher-order themes were created via thematic analysis: gamer motivations, motivation amplifiers, AVG issues, optimistic future. All themes were viewed as important in answering the research questions. For example, to understand why AVGs are not performing well, an understanding of what motivates a gamer to play, and how that motivation is affected, was required. It was also important to understand how AVGs compared to NAVGs in facilitating these motivations. Next, it was important to understand the issues AVGs face from the gamer's perspective. Lastly, by interpreting participants' thoughts on the future of AVGs, potential future directions of AVGs were observed. Figure 2 provides a diagrammatic representation of the created themes. 

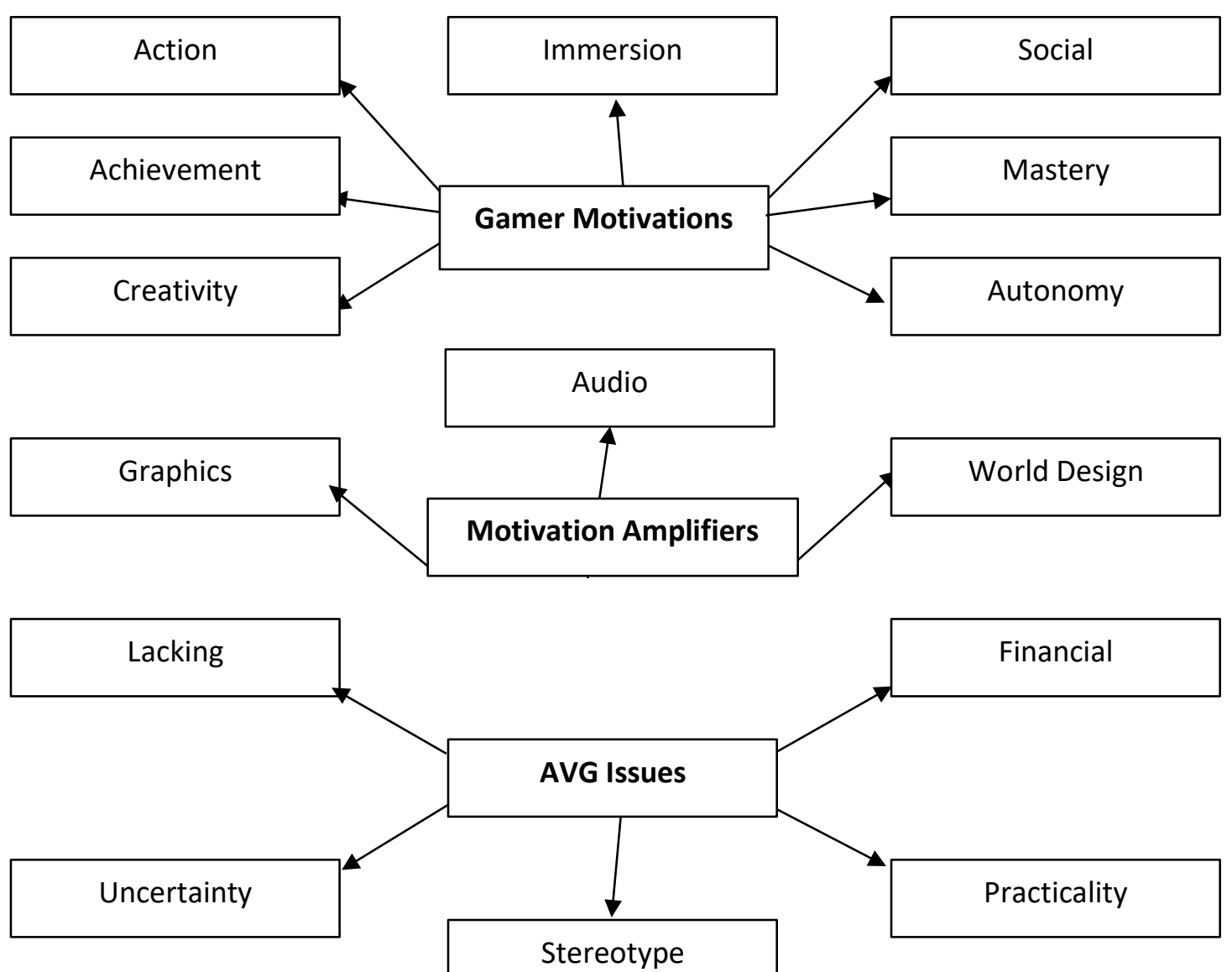

Optimistic Future

Figure 2. Final thematic map. Boldface $=$ Higher-order themes. Lightface $=$ Sub-themes

\subsection{Gamer motivations}

Throughout the interview process, participants frequently mentioned aspects of the gameplay experience that influenced their decisions to play. During refinement of the thematic map, deductive logic was applied by cross-referencing the qualitative data with underpinning theories of this study [28]-[30]. Subsequent findings support previously reported conclusions [28]-[30]. Consequently, sub-themes were labelled to represent links to the GMM [29] and SDT in gaming [30]. The three sub-themes of immersion, autonomy and social were more prominent in the interviews than the remaining four sub-themes of achievement, mastery, action, and creativity. These sub-themes will be discussed next.

\subsection{1: Immersion.}

At the beginning of each interview, to build rapport, participants were asked 'what do video games mean to you?' Each response had immersion as its core, in the sense of being completely absorbed by the game, taking themselves away from their day-to-day living, and going somewhere/being someone else [48]. For example, Evie Frye perceived video games as a form of escapism: 
"That's the sort of thing I like, its escapism. Go somewhere else, see somewhere else. You sit down and you get immersed in it that's the whole point... that's how I see it... that's how see the entertainment thing of it all. It's that living, breathing world."

Geralt of Rivia added "I don't want to be paying attention to the real world or whatever, I tend to go on a game, it sucks me in. Escapism for me in video games is... one of the best qualities."

Immersion was deemed an important aspect of motivation for these participants. However, it is not clear how immersion impacts motivation with regards to AVGs. Geralt of Rivia suggests AVGs do not elicit immersion: "active games... no immersion, engagement or owt. I can play it, there's still real-world problems, there's nowt. There is still things that bother me, some things I might still be thinking about, there is nowt there." Geralt then compares AVGs to NAVGs by saying "Whereas non-active games, it's a completely different world for me." Viktor Reznoz supports this argument:

"I've not found an active game yet where the immersion has been there altogether and its carried on and I've not got bored... but I played a lot of NAVGs where I've played for hours and hours and uhh... being immersed like over a long period of time, like Runescape, I racked up a lot of hours, not even going to say hours, weeks of gameplay on that."

These findings, combined with stage 1, enhance our understanding of how AVGs compare to NAVGs. They also offer partial support for the GMM, as immersion was identified as one of the six main themes influencing gamer motivations. The magnitude of influence is evident, as findings reveal that the inability of AVGs to elicit immersion results in boredom, and the consequential decision to stop playing.

\subsection{2: Autonomy.}

The ability to make choices with far-reaching consequences was perceived as important by participants. Evie Frye values choice, and consequence, noting "It was not the artwork, you felt part of it because you were doing stuff and having an effect, you mattered". Rico Rodriguez provides further support for autonomy:

"... an actual story that has consequences, choices, part of good storytelling is that your actions have consequences and could ultimately affect the end result...for me immersion means... that your actions... influence...the story, the environment... just... alter the gameplay somehow. ...that's what makes games really immersive for me, you can make choices, and they actually have consequences. It's a really big thing for me."

Autonomy was also applied in video games through personal expression and customisation. However, participants viewed customisation as good, but not necessary, provided the ability to express yourself is available through other means (e.g., the choices affecting narrative and environment). Geralt of Rivia suggests:

"I do believe it should be there because it allows people to be creative, to express themselves in an online environment, you can use a different toolset to that they're used to using, so it's cool that they allow them that expression whereas in different types of games it is not really needed, you can express yourself a different way rather than how you look."

These findings provide contrasting support for the GMM and SDT in gaming. Expression and customisation form part of the creativity construct of the GMM. However, customisation was viewed by participants as a good addition rather than a gaming essential. The GMM does not explicitly state the importance of autonomy in gamer motivations, whereas it is a principal component of SDT. Beyond gaming, autonomy, in addition to competence and relatedness, are viewed as facilitators to intrinsic motivation [49], social development [50], and wellbeing [51]. Therefore, the GMM may be excluding a pivotal component of gamer motivation. However, the argument could be made that autonomy 
pag. 12

underpins each game design construct in the GMM, providing freedom to be more specific in identifying gamer motivations.

While autonomy is evidently important, it is unclear how the "choice architecture" (i.e., how the choice is presented, and the subsequent impact on consumer decision-making) [52] is applied in AVGs, in contrast to NAVGs. Rico Rodriguez offers his thoughts on the contrast: "...that is like really immersive like you can... what you do actually affects the game. Whereas active games just seem like they're on rails the entire time. You can't deviate far from that at all." It is difficult to ascertain if a lack of autonomy results in the gamer choosing not to play that game. However, there are many quotes that link autonomy to immersion, as shown in this study and others [29], [48], to influence that decision. This insight aids understanding of why AVGs are not performing well in the market and how they compare to NAVGs.

\subsection{3: Social.}

Being social with other gamers was viewed as important by participants. Two prominent perspectives were identified, the first being community-based, having people to talk with, play with, engage with in some way. Geralt of Rivia states "single player games are surprising because they have communities, they have forums where people talk about the story... characters... the fan fiction, they have it all, they go for ages." Viktor Reznov approaches the community perspective slightly differently:

“...I used to go to computer club and play Runescape together. Used to be like 20 people all there playing Runescape or another game, cause it was a free computer club where you could go and talk to your friends. It was great. That's how I met all my friends actually."

The second perspective was competition-based, achieved through others. This was split between leaderboards/scores, local competition, and online competition. The general perception was that AVGs were fun for local multiplayer, while NAVGs were better for online multiplayer. This was demonstrated by Captain Price:

“...yeah when stuff is done online, it can get competitive, but it's just a voice...whereas in person that's when you start insulting each other, that's when you start getting in your face laughing at you, then you get more worked up and more competitive, so I think that is just like that..."

This idea that AVGs were great for local multiplayer was contrasted by the perception that AVGs were not great at offering online outlets for competition and community. Rico Rodriguez suggests:

“...with active games, it's kind of hard because you need a multiplayer aspect to engage with more people. Yes, you can have like online scoreboards and stuff like that, then it's just a high score thing it's not really a community because, for it to be a community you need to be able to interact with each other and talk or whatnot, whereas I can 't really see a, well I haven't seen an umm... situational game where that happens with active games at the moment."

The importance of social aspects in a video game are demonstrated in positive ways "...online games have longer longevity because they have that community, that social aspect" and negative ways "Main reason I get bored is just because I don't have anyone to play with online that I know and can speak to... like... just boring... it's a lot more fun when you play with other people really."

These findings provide supporting evidence for SDT in gaming, by highlighting the importance of having people to connect with, termed relatedness. While this was found to be true, the findings from this study drew more parallels with the GMMs construct of social, which also refines into community and competitive. Again, this highlights some of the 
overlap between the two key theories alluded to in section 1.4. Nevertheless, the findings from this study provide supporting evidence for what is known regarding the social aspects of gaming.

While supporting current research in the field, this paper also provides novel insights, particularly regarding perceived differences between AVGs and NAVGs. AVGs are perceived as being fun to play from a local multiplayer perspective, but lack the depth when multiplayer goes online. This is a critical point which could affect the total reach of AVGs, given that the online gamer count is 2.5 billion [12]. Thus, AVGs may be able to leverage online to reach more people. However, it could also be an indication of overall game quality. For example, Captain Price stated "I'd play in a group with Wii Sports." He was then asked if he would play on his own. He replied with "no, just see no point." This was representative of the other participants. If the lack of depth in social, particularly online, is a lack of quality, then these findings reinforce those from stage 1.

Collectively, data from stage 1 and stage 2 has helped identify what aspects motivate a person to play games. In other words, the previous sections of this manuscript (namely the quantitative data in section 2.3.1 and the qualitative data within the current section) offer a unique approach to the triangulation of gamer motivation. These findings offer evidence in support of leading motivation theories, such as SDT and the GMM. The findings also contribute to the body of knowledge by identifying comparisons between AVGs and NAVGs.

\subsection{Motivation amplifiers}

Three sub-themes of world design, graphics, and audio were identified. World design had passive and active aspects; passive being how expansive the world was, active being what you could do with the world, the story. Rico Rodriguez talks about the decision-making process in buying a game:

"World design definitely for me is one... that's what initially grabs me... that's what you see in all the advertisements and stuff like that is the world design. And then quite often seems to be the case, granted not all the time but if... a developers spent a lot of time making the world look as a good as it does... they more than likely spend the same amount of time if not more on the characters and the story so... that seems to be as like... an initial peek my interest, is the world design."

Rico goes on to state: "buy it for the world design then look at what you can do with the world, is one of the things that draws me into that game."

Evie Frye, after being asked about their worst moments in games, began to talk about the graphics of a recent game purchase stating:

"Okay, we are in 2016 and your graphics look like something that came out of 2008... I get excited when I see a game and it looks... absolutely spectacular. Because then I know that's when someone sat there and poured their heart and soul into... because that's what I'm going to do now."

Referring to the capabilities of audio, Captain Price adds "yeah you want that feeling, if you're playing a horror game, you want that feeling of suspense, like something is going to pop out on you, you're terrified...". The critical point here is not the mere mention of the three sub-themes, rather the effect each has. For example, world design was enough to influence Rico Rodriguez to purchase. The quality of the audio facilitated the feeling of suspense and terror in Captain Price. The quality of the graphics was considered important enough for Evie Frye to associate previous game choice with their best/worst moments in gaming.

It seems, from these quotes, and the references to world design, graphics, and audio in game design textbooks [31], [32], that these design aspects greatly influence gamer 
pag. 14

motivation. However, the design aspects were not included in any of the authors' underpinning theories of gamer motivation [28]-[30]. One possible explanation is that, while world design, graphics, and audio do have an impact on gameplay experience, they are not essential to the gamer's motivation. For example, Viktor Reznov suggests "Runescape is not really a graphically good game, and the audio on it is terrible, it's weird how...I racked up...weeks of gameplay on that.". Rico Rodriguez adds:

“...Minecraft, it's got 64-bit graphics for Pete's sake, but it's still fun, that's one of the things like... if you get the gameplay down, like it's a fun game to play and it's constantly evolving, you can figure out new stuff to do then... the... graphics can take a little bit of a hit."

Rico also suggests "if the game's unplayable... then it's not worth it. You won't get anything out of it, I just spent my money on nothing [even]...If it looked fantastic and everything but the gameplay was shit."

Findings from stage 1 showed that world design is viewed as worse than the genre mean. Graphics and audio, while above the genre mean, could still be viewed as subjectively poor. In conjunction with stage 2 data, the implication is that a lack of focus is given to applying the GMM, or to developing world design, graphics, and audio in AVGs. Regardless, the indication is that an overall lack of quality is evident.

On balance, these findings suggest that if world design, graphics, and audio are executed well, they may have an amplifying effect on gamer motivation, when in conjunction with aspects of the GMM. Thus, world design, graphics and audio appear less relevant than the GMM, with regards to motivation. Consequently, when developing games, particularly under budget and time constraints, it may be more prudent to focus on applying the GMM.

\subsection{AVG issues}

There were five sub-themes within AVG issues. "Lacking" and "practicality" were prominent and will be discussed in this section. A third prominent (yet speculative) subtheme was "financial", including reference to budgets and investment, information which is limited in accessibility and scope for public scrutiny. Given that participants were consumers only, financial issues will not be discussed in this section. The remaining two sub-themes - "stereotype" and "uncertainty" - were under-represented in the data with one and three quotes, respectively. However, there is potential importance that should be explored. For example, regarding stereotypes, Geralt of Rivia states:

"I feel the big stereotype in active games is that umm... it's more for your casuals, your families, your kids so I feel like that stereotype heavily influences... any form of like... Esports organisations or events picking it up because of the stereotype, they don't want the laid-back gamers, they want people who are willing to invest their time into a game, serious enough to do it as a job. I don't think the active games has it..."

Should this opinion be representative of an organisation's thought processes, then AVGs have an issue that requires investigation. Business partnerships are one of the most effective ways to manage technological turbulence and dynamic market environments [53]. The issue could be worsened, should the 'it's not for me' stereotype be representative of the wider population, particularly if that's how consumers think when deciding to purchase a game. Both sides of the issue require further exploration.

\subsection{1: Lacking.}

Participants shared a sense that, at some point soon, the games community will have disappeared. Geralt of Rivia states: 'I don't want to invest into it because I don't know the community will stick around for it but in terms of other examples for active games. I feel like the community is dead, if that." Later adding "... if there is no communication between developers and you and there is no real sense of people playing it then what is the point of 
sticking around." This uncertainty seems to supplement a larger issue of overall quality, implicated in stage 1, forming part of the most prominent sub-theme of lacking. Fundamental issues were the limited quality, depth, and variety of AVGs.

Regarding the quality of AVGs, Viktor Reznov states: "Wii Sports was, it's quite bad, it's just a couple of Miis that had like weird ball bodies and little stumpy arms". Evie Frye adds: "you ended up with the bare minimum. And the game like Star Wars suffered for it. Where you could go into the world... it was limited... because of what it could do. So... I didn't find it very good." Geralt of Rivia continues: "for me, active games, there is nowt there. Its crap, that's one way of putting it really." Captain Price, discussing the graphics of AVGs: "yeah it delivers, its fine for like Wii Sports, Just Dance, the graphics it has are fine, but I still just think they're terrible, compared." Lastly, Rico Rodriguez summarises his thoughts:

“...AVGs don't really have that initial pull. It's like oh here's a generic AVG, oh you're a ninja this time... these are your enemies. It's that sort of thing, it almost seems like a cut and paste, just changing something on a slider..."

Without doubt, these participants view AVGs as lacking quality. However, what was viewed as a lack of quality, may, in fact, be a lack of depth. There are instances of better experiences, where the participants enjoy the AVG for a short while before ultimately deciding they lack quality. For example, Viktor Reznov thinks: "Pokémon Go is... it was good but I just got bored quite quickly", while Geralt of Rivia adds: "I think it's just a big farce, the active games, they're fun for a while don't get me wrong but umm... I don't think they have the depth that non-active ones do." Captain Price bolsters the argument: "when the Wii got actually released, everyone was all over it and it was like oh yes this is brilliant, couple of months down the line it all dies out, becomes a fad of the past". This could be indicative of a novelty aspect, showing that gamers are eager to try something new, something active. However, it could also reflect a general lack of quality, or a combination of the two. Regardless, this issue is worthy of further exploration.

Issues of longevity within video games have always been evident [31], [32]. Cequently, authors of game design textbooks often discuss how to keep people playing their games [31], [32]. The general consensus from participants was that AVGs lack variety. Captain Price suggests: "there isn't a wide variety to be fair, but not many come with a story anyway so...". Evie Frye states: "I always thought... there was never enough games out there for adults." Geralt of Rivia summarises this lack of choice:

"I feel their ideas and their... they're too closed minded, I think they need to become a bit more expressive, outside the box type, because I mean if one person can make a game and sell millions, even if its a pixel based game, it captivated hundreds of thousands of people, I don't see why an active game couldn't do that. I really feel like they just need to think outside of the box rather than the typical tropes of games. Maybe try captivating a wider audience, maybe try pull them rather than just say this is for mum and dad and kids and that's it leave it at that."

With the variety of gamer motivations represented by the GMM, it seems plausible that one good AVG would not shift perceptions, even with quality and depth. Perhaps multiple games, spanning multiple motivations, are required for AVGs to be considered successful and to compete with NAVGs.

These findings, particularly regarding the quality of AVGs, supplement those from stage 1 , which also suggest a lack of quality in AVGs compared to NAVGs. These findings raise important questions that require further exploration, including how the stereotype of "AVGs are for kids and families' might affect the development of strategic business partnerships or the gamer's decision to buy. Such views could be interpreted as either a prejudiced view of AVGs, a prejudiced view of kids and families, or both. This is crucial to note, given that the themes and quotes reported within this paper represent the authors' efforts to 
pag. 16

authentically reflect the views of those who participated in the study; a broader representation of such stereotypes requires extended enquiry beyond the scope of this study. In addition to exploring methods to bridge the gap in quality between AVGs and NAVGs, these findings suggest there may be a novelty aspect to AVGs. There have been explorations into the human need for novelty as a powerful motivator [54]. This outlines potential for the AVG genre to capitalise on, should novelty be effectively combined with excellent quality and expansive depth, leading to longer-term engagement.

\subsection{2: Practicality.}

Since the key feature of AVGs is motion detection, there is a requirement for additional technology, beyond the normality of console, mobile, or computer. Participants viewed current technology as limited in terms of practicality. Focusing on practicality at home, Evie Frye suggests: "I think that's the whole problem. You need a really big room." Rico Rodriguez adds: "It was just a pain in the arse to set up, you had a certain space for it to work, that space had to be clear, you had to sync yourself to it, so many impractical problems".

Moreover, there are limitations in hardware performance, as Viktor Reznov discusses mobile AVGs: "the battery drain was quite annoying, so that ruined it a little bit, I'm guessing that's a problem, if you're outside I'm guessing you will use your phone, the amount of battery it uses is astounding". Rico Rodriguez analysed Kinect: "It's not finetuned enough to do like, oh you can't move your arm slightly different or in a different position. It has to be drastically different motions for it to be able to pick up."

More practical limitations are evident within the gameplay. For example, Captain Price states: "...they could easily throw a storyline in, amateur dancing, work your way up, but the dancing might take away from it". Rico Rodriguez adds: "for a game to be active, you need to be moving, pretty much constantly". This poses a difficult problem; for an AVG to be active, you need to be moving, but the movement itself could take away from the immersive qualities of traditional video games, thus affecting perceived quality.

These findings suggest a need for technological advancement or innovation with a focus on retaining the unique, intrinsically-motivating pull of NAVGs, in-line with the GMM. This section, regarding AVG issues, further emphasises the difference between AVGs and NAVGs in terms of quality. It also develops the understanding of why AVGs are not performing well on the market, by highlighting key issues; lack of perceived quality and variety, the stereotype, and limited practicality, should be explored in future research.

\subsection{Optimistic future}

Initially, with Evie Frye, there was a past tense to the interview, implying that AVGs had been and gone. However, throughout the remainder of interview (and indeed those of other participants), the tone changed. There was optimism, hope, but perhaps more importantly, functional potential. For example, base hope from Evie Frye: "I think it will get there eventually. Hopefully..." and Captain Price: "I remain hopeful that something active is built into RPG. Hoping that something like that sword art online happens in the real world, I would be all over that". Viktor Reznov, exploring the functional potential of augmented reality, adds: “... acting like I've got a $2 \mathrm{H}$ in my hand, swinging at trees, I think Minecraft would be quite cool, outside somehow, hitting trees with your fist, or your phone". Victor went on:

"I suppose if you could... sort of uhh... interact with other gamers... through the storyline... say if you had like a big dragon boss battle and you could recruit your friends and all meet up... I wonder if they could hire actors for the AVGs, and their purpose in the video game is to just uhh give out missions and stuff..." 
Throughout the interviews, early reflection indicated that the perceived lack of quality and practical limitations could be prominent throughout. This led to the development of an idea to address these issues. This idea was added to the interview guide to gauge perception.

The idea was described to participants with some variation of the following: You have three categories of physical stats - strength, speed and endurance. Each category has a maximum level of 100 and has 5-10 exercises per category (e.g., barbell squat, 100m sprint, $5 \mathrm{k}$ run). Physical performance in these categories, in reality, results in a level being attributed to your avatar, digitally, which is on a central platform. Your avatar's stats, for example, could be 83 strength, 87 endurance and 93 speed. These stats can then be downloaded into the video games character and played with, in whichever video game you wanted.

Overall, responses to the above idea were positive. All five participants wanted to try the platform. Two participants who currently exercised said they would use the platform if it could be used concurrently with their exercise routine. Two physically-inactive participants (i.e., categorised as such by the fact that they did not currently meet the recommended weekly amount of physical activity for adults [19]) were asked if they would start exercising to develop their avatar's stats. Rico Rodriguez answered simply with "yeah I would, if the gameplay is based around your physical attributes." Whereas Captain Price answered with:

"Yeah I'd go for it. I'd definitely try it. I mean... yeah... your improving your own physical health, there's a lot of achievement there, I mean you're improving yourself in real life and you're improving your character in the game... you have a balance between playing the game and not playing the game. Improving yourself so that you can eventually... p... play the game even more, play even better."

The fifth participant manoeuvered away from a committed answer and opted to discuss gamers' social anxiety as a consideration. These findings take the line of inquiry one step further by offering a plausible, practical solution to previously discussed issues.

\subsection{Limitations}

Limitations were evident in the sample regarding sex, occupation, ethnicity, and socioeconomic status. First, GameTrack [13] shows that the UK gamer population is $42 \%$ female, while the sample in this study was $22 \%$ female. Furthermore, no female participants took part in stage 2. It is possible that valuable data were missed. Future research should aim to build a more authentic population sample, according to reputable video game industry statistics [14]. Second, it became evident throughout the interview process, wherein participants were quoting low budgets and funding issues, that opinions from a more specialised sample would have been beneficial to the research problem. Considering this point, future research should explore the research problem from more specialised samples, such as; game developers, academics, retail specialists, and in general, industry experts. Third, data were not collected on participants' ethnicity or socioeconomic status, an oversight of the authors. Nesta [55] stated that ethnicity or economic background does not influence the decision to play games. However, it is undetermined whether ethnicity or socioeconomic status may influence the perceptions of gameplay experience, particularly regarding AVGs. Thus, future research should consider the research problem with these variables in mind. Finally, the authors acknowledge that there are myriad exciting areas for further exploration which were, unfortunately, beyond the scope of this study. Such important elements including game mechanics [56], [57], reality-enhanced gaming [58] and cultural considerations aligned to the importance of reality and video game addiction, a current and fiercely contested topic [59]-[62], are worthy of further scrutiny.

\subsection{Future directions and implications}


pag. 18

Future research should replicate the study to increase the sample size so that the demographics can be balanced out and compared, while including expert input. Several AVG issues - lack of quality and variety, the stereotype, and practicality - were identified through qualitative exploration and should be explored further. A proposed mixed-reality platform was viewed positively by participants, offering a plausible and practical solution to the AVG paradox which should be further explored, initially with a large sample, then with a specialised sample, and finally, in a controlled intervention setting.

There was one prominent implication for practice: findings supported the GMM, more than SDT. Given the reported differences in the perceptions of the gameplay experiences between AVGs and NAVGs, AVG developers should focus on applying the GMM to their game development. If the GMM has been applied, and there are resources remaining prior to finishing the game, AVG developers should focus on increasing the quality of the game's world design, graphics, and audio.

A final pertinent question (posed by one of the reviewers of this manuscript) is worth considering: "Is the lower quality of AVGs something intrinsic or substantially a matter of budget?". Given that gaming in its entirety represents a multi-billion dollar industry on a global scale, the authors urge fellow researchers to explore this question in greater depth. It is hoped and envisaged that the financial resources made available on a regular basis for the development of NAVGs could be harnessed for the development of entertaining, motivating, and commercially successful AVGs, which have the potential to reap a range of health benefits.

\section{Conclusion}

The purpose of this study was to explore why AVGs are not performing well in the market, and how they compare to NAVGs. To the authors' knowledge, this was the first time such a study had been undertaken, the findings of which represent a novel and meaningful contribution to the science of gaming. Participant data revealed that AVGs are perceived to be significantly worse than NAVGs, when measured for gameplay experience. This finding was consistent across quantitative and qualitative methods, signalling the first step in understanding why AVGs are not performing well on the market. Rigorous qualitative analysis highlighted several issues that require further exploration through future scientific enquiry. Primary issues included the lack of quality and variety in AVGs, the stereotype that AVGs were just for kids and families, and practical limitations of AVG hardware.

Underpinning the decision to play and keep playing was gamer motivation. Findings support the GMM, which AVG developers are recommended to apply. Where time and budget allow, developers should focus on increasing the quality of world design, graphics, and audio of the game, which operate as motivation amplifiers. The central idea of AVGs, wherein you can play and get fitter, was a meaningful one to participants, and despite discrepancies in perceived quality, participants remained hopeful that the AVG genre will improve. Participants lauded a mixed-reality platform idea, with all keen to try it. This idea presents a unique yet plausible, practical solution to several of the issues discussed, and should be further explored. The perspective that AVGs compete with NAVGs for a players attention is a vital contribution to the scientific literature. Future AVG research should assess AVGs for entertainment/engagement compared to NAVGs, in addition to the standard pragmatic usefulness of the intended, and deliverable effect of the game. In conclusion, the reason why AVGs perform poorly on the market is multi-faceted...but not without hope. 


\section{References}

[1] D. Ding et al., "The economic burden of physical inactivity: a global analysis of major noncommunicable diseases", The Lancet, vol. 388, no. 10051, pp. 1311-1324, 2016. Available: https://doi.org/10.1016/S0140-6736(16)30383-X

[2] J. Knight, "Physical inactivity: Associated diseases and disorders", Annals of Clinical \& Laboratory Science, vol. 42, no. 3, pp. 320-337, 2012. Available: http://www.annclinlabsci.org/content/42/3/320

[3] Harvard School of Public Health, "The Global Economic Burden of Non-Communicable Diseases", World Economic Forum, 2011.

[4] Chartered Institute of Personnel and Development, "Absence Management", 2016.

[5] PwC, "The Rising Cost of Absence", 2013.

[6] International Sport and Culture Association \& Centre for Economics and Business Research, "The Economic Cost of Physical Inactivity in Europe", 2015.

[7] M. Chinapaw, K. Proper, J. Brug, W. van Mechelen and A. Singh, "Relationship between young peoples' sedentary behaviour and biomedical health indicators: a systematic review of prospective studies", Obesity Reviews, vol. 12, no. 7, pp. e621-e632, 2011. Available: https://doi.org/10.1111/j.1467-789X.2011.00865.x

[8] McKinsey Global Institute, "How the world could better fight obesity", 2014

[9] R. Rhodes, R. Mark and C. Temmel, "Adult Sedentary Behavior", American Journal of Preventive Medicine, vol. 42, no. 3, pp. e3-e28, 2012. Available: https://doi.org/10.1016/j.amepre.2011.10.020

[10] A. Thorp, N. Owen, M. Neuhaus and D. Dunstan, "Sedentary Behaviors and Subsequent Health Outcomes in Adults", American Journal of Preventive Medicine, vol. 41, no. 2, pp. 207-215, 2011. Available: https://doi.org/10.1016/j.amepre.2011.05.004

[11] M. Tremblay et al., "Systematic review of sedentary behaviour and health indicators in schoolaged children and youth", International Journal of Behavioral Nutrition and Physical Activity, vol. 8, no. 1, p. 98, 2011. Available: https://doi.org/10.1186/1479-5868-8-98

[12] Newzoo, "Global games market report", Newzoo, 2019.

[13] GameTrack, "GameTrack digest", 2016.

[14] The Association for UK Interactive Entertainment, "Gaming Revolution Infographic", 2016.

[15] L. Larsen, L. Schou, H. Lund and H. Langberg, "The Physical Effect of Exergames in Healthy Elderly-A Systematic Review", Games for Health Journal, vol. 2, no. 4, pp. 205-212, 2013. Available: https://doi.org/10.1089/g4h.2013.0036

[16] A. Lu, H. Kharrazi, F. Gharghabi and D. Thompson, "A Systematic Review of Health Videogames on Childhood Obesity Prevention and Intervention", Games for Health Journal, vol. 2, no. 3, pp. 131-141, 2013. Available: https://doi.org/10.1089/g4h.2013.0025

[17] W. Peng, J. Crouse and J. Lin, "Using Active Video Games for Physical Activity Promotion", Health Education \& Behavior, vol. 40, no. 2, pp. 171-192, 2012. Available: https://doi.org/10.1177/1090198112444956

[18] J. van 't Riet, R. Crutzen and A. Lu, "How Effective Are Active Videogames Among the Young and the Old? Adding Meta-analyses to Two Recent Systematic Reviews", Games for Health Journal, vol. 3, no. 5, pp. 311-318, 2014. Available: https://doi.org/10.1089/g4h.2014.0005

[19] World Health Organisation, "Global recommendations on physical activity for health", 2010.

[20] "Global yearly video game chart", VGChartz, 2012. [Online]. Available: http://www.vgchartz.com/yearly/2012/Global/

[21] "Global yearly video game chart", VGChartz, 2013. [Online]. Available: http://www.vgchartz.com/yearly/2013/Global/

[22] "Global yearly video game chart", VGChartz, 2014. [Online]. Available: http://www.vgchartz.com/yearly/2014/Global/

[23] "Global yearly video game chart", VGChartz, 2015. [Online]. Available: http://www.vgchartz.com/yearly/2015/Global/

[24] "Global yearly video game chart", VGChartz, 2016. [Online]. Available: http://www.vgchartz.com/yearly/2016/Global/

[25] "Global yearly video game chart", VGChartz, 2017. [Online]. Available: http://www.vgchartz.com/yearly/2017/Global/

[26] "Global yearly video game chart", VGChartz, 2018. [Online]. Available: http://www.vgchartz.com/yearly/2018/Global/ 
[27] E. Deci and R. Ryan, "Self-Determination", The Corsini Encyclopedia of Psychology, 2010. Available: https://doi.org/10.1002/9780470479216.corpsy0834

[28] S. Rigby and R. Ryan, Glued to games. Santa Barbara, Calif.: ABC-CLIO, 2011.

[29] N. Yee and N. Ducheneaut, "Our Gamer Motivation Model", Quantic Foundry, 2019. [Online]. Available: https://quanticfoundry.com/

[30] R. Ryan, C. Rigby and A. Przybylski, "The Motivational Pull of Video Games: A SelfDetermination Theory Approach", Motivation and Emotion, vol. 30, no. 4, pp. 344-360, 2006. Available: https://doi.org/10.1007/s11031-006-9051-8

[31] E. Adams, Fundamentals of game design. [Berkeley, CA]: New Riders, 2014.

[32] M. Salmond, Video Game Design. [S.L.]: Bloomsbury Publishing, 2019.

[33] "Game section", Metacritic, 2017. [Online]. Available: http://www.metacritic.com/game.

[34] T. Tofade, J. Elsner and S. Haines, "Best Practice Strategies for Effective Use of Questions as a Teaching Tool", American Journal of Pharmaceutical Education, vol. 77, no. 7, p. 155, 2013. Available: https://doi.org/10.5688/ajpe777155

[35] A. Sparkes and B. Smith, Qualitative research methods in sport, exercise and health. London: Routledge, 2014.

[36] "Pokemon Go", Metacritic, $2017 . \quad$ [Online]. Available: http://www.metacritic.com/game/ios/pokemon-go. [Accessed: 11- Nov- 2017].

[37] "Just Dance 2017", Metacritic, 2017. [Online]. Available: http://www.metacritic.com/game/xbox-one/just-dance-2017. [Accessed: 11- Nov- 2017].

[38] "Wii Fit", Metacritic, 2017. [Online]. Available: http://www.metacritic.com/game/wii/wii-fitplus. [Accessed: 11- Nov- 2017].

[39] "Wii Sports", Metacritic, 2017. [Online]. Available: http://www.metacritic.com/game/wii/wiisports. [Accessed: 11- Nov- 2017].

[40] "Kung Fu Live", Metacritic, 2017. [Online]. Available: http://www.metacritic.com/game/playstation-3/kung-fu-live. [Accessed: 11- Nov- 2017].

[41] "Beatsplosion", Metacritic, 2017. [Online]. Available: http://www.metacritic.com/game/xboxone/beatsplosion-for-kinect. [Accessed: 11- Nov- 2017].

[42] J. Dewey, J. Boydston and L. Hickman, The Collected Works of John Dewey, 1882-1953 (2nd release). Electronic edition. The Middle Works of John Dewey, 1899-1924. Volume 4. Charlottesville, Va.: InteLex Corporation, 1996.

[43] J. Dewey, J. Boydston and L. Hickman, The Collected Works of John Dewey, 1882-1953 (2nd release). Electronic edition. The Middle Works of John Dewey, 1899-1924. Volume 12. Charlottesville, Va.: InteLex Corporation, 1996.

[44] D. Morgan, "Paradigms Lost and Pragmatism Regained", Journal of Mixed Methods Research, vol. 1, no. 1, pp. 48-76, 2007. Available: https://doi.org/10.1177/2345678906292462

[45] D. Morgan, "Pragmatism as a Paradigm for Social Research", Qualitative Inquiry, vol. 20, no. 8, pp. 1045-1053, 2014. Available: https://doi.org/10.1177/1077800413513733

[46] J. Saldaña, "Blue-Collar Qualitative Research", Qualitative Inquiry, vol. 20, no. 8, pp. 976-980, 2014. Available: https://doi.org/10.1177/1077800413513739

[47] V. Braun and V. Clarke, "Using thematic analysis in psychology", Qualitative Research in Psychology, vol. 3, no. 2, pp. 77-101, 2006. Available: https://doi.org/10.1191/1478088706qp063oa

[48] E. Boyle, T. Connolly, T. Hainey and J. Boyle, "Engagement in digital entertainment games: A systematic review", Computers in Human Behavior, vol. 28, no. 3, pp. 771-780, 2012. Available: https://doi.org/10.1016/j.chb.2011.11.020

[49] E. Deci and R. Ryan, "The "What" and "Why" of Goal Pursuits: Human Needs and the SelfDetermination of Behavior", Psychological Inquiry, vol. 11, no. 4, pp. 227-268, 2000. Available: https://doi.org/10.1207/S15327965PLI1104_01

[50] E. Deci and R. Ryan, Intrinsic motivation and self-determination in human behavior. New York: Springer Science+Business Media, 2014.

[51] J. Chang, C. Huang and Y. Lin, "Mindfulness, Basic Psychological Needs Fulfillment, and Well-Being", Journal of Happiness Studies, vol. 16, no. 5, pp. 1149-1162, 2014. Available: https://doi.org/10.1007/s10902-014-9551-2

[52] R. Thaler, C. Sunstein and S. Pratt, Nudge. [United States]: Gildan Audio, 2009.

[53] W. Jiang, Business Partnerships and Organizational Performance. Berlin, Heidelberg: Springer Berlin Heidelberg, 2014.

[54] W. Gallagher, New. New York: Penguin Books, 2013. 
[55] "Did you really take a hit? Understanding how video games playing affects individuals", Nesta, 2017. [Online]. Available: https://www.nesta.org.uk/report/did-you-really-take-a-hitunderstanding-how-video-games-playing-affects-individuals/.

[56] S. Arnab, T. Lim, M. B. Carvalho, F. Bellotti, S. de Freitas, S. Louchart, N. Suttie, R. Berta, A. De Gloria, "Mapping learning and game mechanics for serious games analysis", British Journal of Educational Technology, vol. 46, no. 2, pp. 391-411, 2014. Available: https://doi.org/10.1111/bjet.12113

[57] M. Carvalho, F. Bellotti, R. Berta, A. De Gloria, C. Islas Sedano, J. Baalsrud Hauge, J. Hu, and M. Rauterberg, "An activity theory-based model for serious games analysis and conceptual design", Computers \& Education, vol. 87, pp. 166-181, 2015. Available: https://doi.org/10.1016/j.compedu.2015.03.023

[58] R. Massoud, S. Poslad, F. Bellotti, R. Berta, K. Mehran and A. De Gloria, "A Fuzzy Logic Module to Estimate a Driver's Fuel Consumption for Reality-Enhanced Serious Games", International Journal of Serious Games, vol. 5, no. 4, pp. 45-62, 2018. Available: https://doi.org/10.17083/ijsg.v5i4.266

[59] C. Ferguson, A. Bean, R. Nielsen and M. Smyth, "Policy on unreliable game addiction diagnoses puts the cart before the horse.", Psychology of Popular Media Culture, 2019. Available: https://doi.org/10.1037/ppm0000249

[60] C. Ferguson and J. Colwell, "Lack of consensus among scholars on the issue of video game "addiction".", Psychology of Popular Media Culture, 2019. Available: https://doi.org/10.1037/ppm0000243

[61] D. Kuss, M. Griffiths and H. Pontes, "DSM-5 diagnosis of Internet Gaming Disorder: Some ways forward in overcoming issues and concerns in the gaming studies field", Journal of Behavioral Addictions, vol. 6, no. 2, pp. 133-141, 2017. Available: https://doi.org/10.1556/2006.6.2017.032

[62] M. Sprong, M. Griffiths, D. Lloyd, E. Paul and F. Buono, "Comparison of the Video Game Functional Assessment-Revised (VGFA-R) and Internet Gaming Disorder Test (IGD20)", Frontiers in Psychology, vol. 10, 2019. Available: https://doi.org/10.3389/fpsyg.2019.00310 